\title{
Predicting Battery Charge Depletion in Wireless Sensor Networks Using Received Signal Strength Indicator
}

\author{
Inacio Henrique Yano, Vitor ChavesDe Oliveira, \\ Eric Alberto de Mello Fagotto, Alexandre De Assis Mota and Lia Toledo Moreira Mota
}

\author{
Department of CEATEC, Electrical Engineering Faculty, \\ Pontifical Catholic University of Campinas, Campinas-São Paulo, CEP 13086-900, Brazil
}

Received 2013-05-16, Revised 2013-05-24; Accepted 2013-06-12

\begin{abstract}
This article aims to identify an adequate mathematical model to predict battery power depletion at the nodes of a Wireless Sensor Network (WSN), by analyzing the Received Signal Strength Indicator (RSSI). Six general models were tested, the simplest Average model, Linear Regression model, Autoregressive (AR) models and Autoregressive Moving Average (ARMA) models. The selected model (AR) presented a low absolute mean residue and adequately represents the charge depletion process, permitting to predict its behavior and to detect the best moment to replace batteries in the WSN nodes.
\end{abstract}

Keywords:RSSI, Battery Discharge, System Identification, Wireless Sensor Networks, Mathematical Model, ARMA

\section{INTRODUCTION}

The Wireless Sensor Networks (WSN) implement the international standard IEEE 802.15.4 (Park et al., 2012) and are usually used in places of difficult access and/or where there is no cabled infrastructure. The independence of cabled infrastructure is simultaneously an advantage and a weak point of a WSN, because it relies on the use of batteries to operate. So, the life cycle of a node/device in a WSN is limited by the battery power capacity. Therefore, it becomes important to predict the sensor's usage duration, before the battery charge depletion occurs (Tong et al., 2011).

The RSSI-Received Signal Strength Indicator (Hellan and Stengel, 2012) is an indicator of this depletion, because it is strongly affected by the battery discharge, when the sensor can no longer send the frames with the power required to transmit in aselected level.

In this scenario, this article presents a study based on the assembly of a practical experiment with a WSN, encompassing data collection, definition and validation of black box mathematical models, with a top down approach (Zhu et al., 2012), to predict when battery charge exhaustion starts in the nodes of a network of wireless sensors by analyzing the variations of the downlink via RSSI.

The relationship between transmit power $(\mathrm{dBm})$ and supply voltage (Volts) is described in (Mafuta et al., 2012).

\subsection{Predicting Battery Charge Depletion}

The usual way to predict battery charge depletion is to watch the supply voltage until a cut-off voltage, based on battery behavior, has been reached (Kerasiotis et al., 2010). Using RSSI instead of supply voltage has some advantages, because it can be read directly from the node's Operating System.Supply voltage needs a voltage divider to be read (Fig. 1), because the Operating System use the supply voltage as a voltage reference (Arduino, 2012a) to make all the measurements at the input pins, so a directly read of battery voltage will always return the same voltage reference, for example $3.3 \mathrm{~V}$ for RFBee (Raioit, 2012; SS, 2013). To get the battery voltage first is necessary to change the voltage reference, for example to $1.1 \mathrm{~V}$ and use a voltage divider to take a voltage not greater than $1.1 \mathrm{~V}$ at the input pin. $\begin{aligned} \text { Corresponding Author: } & \text { Vitor Chaves De Oliveira, Department of CEATEC, Electrical Engineering Faculty, } \\ & \text { Pontifical Catholic University of Campinas-São Paulo, CEP 13086-900, Brazil }\end{aligned}$ 


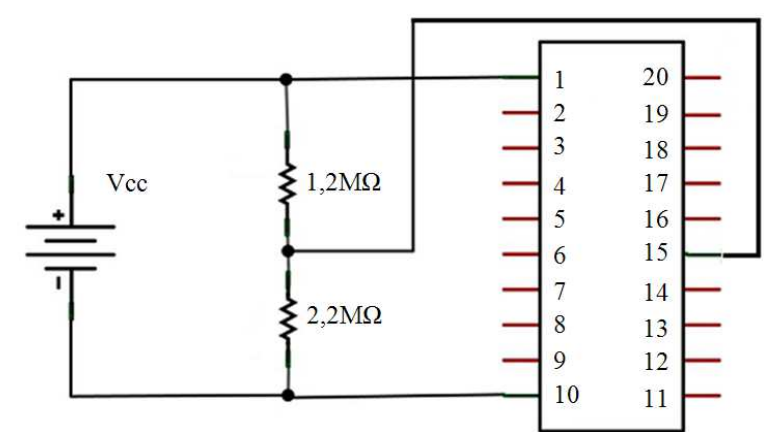

Fig. 1. Voltage divider to read the battery voltage after the voltage reference has been changed

This procedure requires a calibration to get a multiplication factor which will return the real supply voltage value.

After change the voltage reference all the input pins will use the new reference to take the measurements, because of this all of them will need a voltage divider if the transducer voltage exceeds $1.1 \mathrm{~V}$.

Another disadvantage of use a voltage divider is that it will drain current continuously even though the node stays in sleep mode, reducing the node lifetime.

\section{MATERIALS AND METHODS}

In the experimental arrangement, set up to obtain measurements of RSSI seeking to monitor the depletion of the batteries, it was used RFBee v1.1 wireless sensors. This type of WSN sensor (Raioit, 2012; SS, 2013; ATmega, 2012; TI, 2012; Arduino, 2012b) is composed of a microcontroller ATMEGA-168 and a CC1101 transceiverthat allows programming in a language similar to $\mathrm{C}++$, on the same IDE (Integrated Development Environment) of Arduino. That reduces programming complexity when compared to other WSN proprietary platforms.

The measurements and samplings of RSSI were made in aremote wireless sensor that was powered by two AA rechargeable 2500mAh batteries (Fig. 2).

The system identification technique consists of five steps (Zhu et al., 2012): data collection, model type selection, model structure selection, model parameters estimation and model validation. The following models were tested: Average, Linear Regression, Autoregressive (AR), Autoregressive with a constant, Autoregressive Moving-Average (ARMA) and Autoregressive Moving Average (ARMA) of orders 1 and 2. The autoregressive (AR) showed the best results, i.e., presented the smallest residue and is presented in the following section.

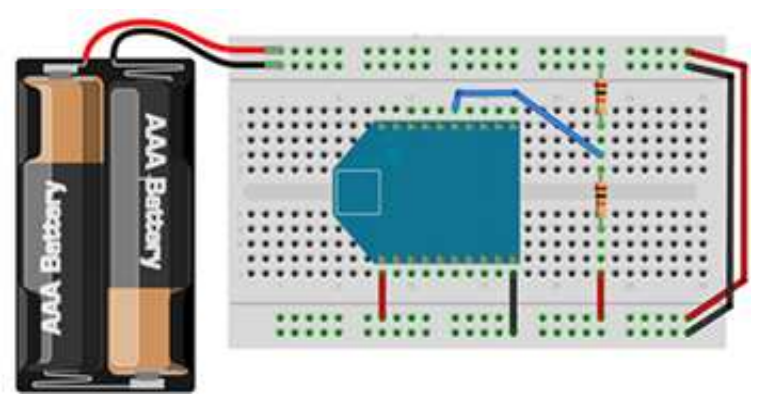

Fig. 2. Remote node where samples of RSSI was collected

This model is only for a static Wireless Sensor Network, since RSSI is affected by distance and the environment where the data was collected had the following characteristics and made in two experiments, the first one was used for system identification and the second one to validate the system in another condition:

- All the transmissionswere made with the maximum power level

- Sampling interval: In first experiment, the time between sampleswas $2 \mathrm{~s}$, this $2 \mathrm{~s}$ were divided into a sleep scheduled time of $1.75 \mathrm{~s}$ and $0.25 \mathrm{~s}$ of activity time. At the second experiment there wasn't sleep time and the node send 4 samples every each second

- Ambient temperature

- Both experiments were made indoor illustrate by Fig. 3. The first collected sampling was at the RN1 (Remote Node 1) orblue node and the second collected sampling was at the RM2 (RemoteNode 2) or the red node. The CB (Central Base) or black node is the sink node

- The experiments were done in two steps and without interference of another network working at the same channel to avoid collisions and frame loss

\subsection{Autoregressive Mathematical Model}

The Autoregressive model (Bourke, 2012) is generally defined by the Equation 1:

$\mathrm{y}_{\mathrm{t}}=\varnothing_{0}+\varnothing_{1} \cdot \mathrm{y}_{\mathrm{t}-1}+\varnothing_{2} \cdot \mathrm{y}_{\mathrm{t}-2}+\ldots+\varnothing_{\mathrm{p}} \cdot \mathrm{y}_{\mathrm{t}-\mathrm{p}}+\varepsilon_{\mathrm{t}}$

Whereas: $y_{t}$ corresponds to the observation of the temporal series at timet; $\phi_{\mathrm{p}}$ corresponds to the parameter of the autoregressive model of order $\mathrm{p}$ and $\varepsilon$ t represents anrandomerror corresponding to random events that can affect the WSN.

The application of the Autoregressive model requires stationarity. This application can be considered when the differentiation of the seriesresults in a stationary series. 


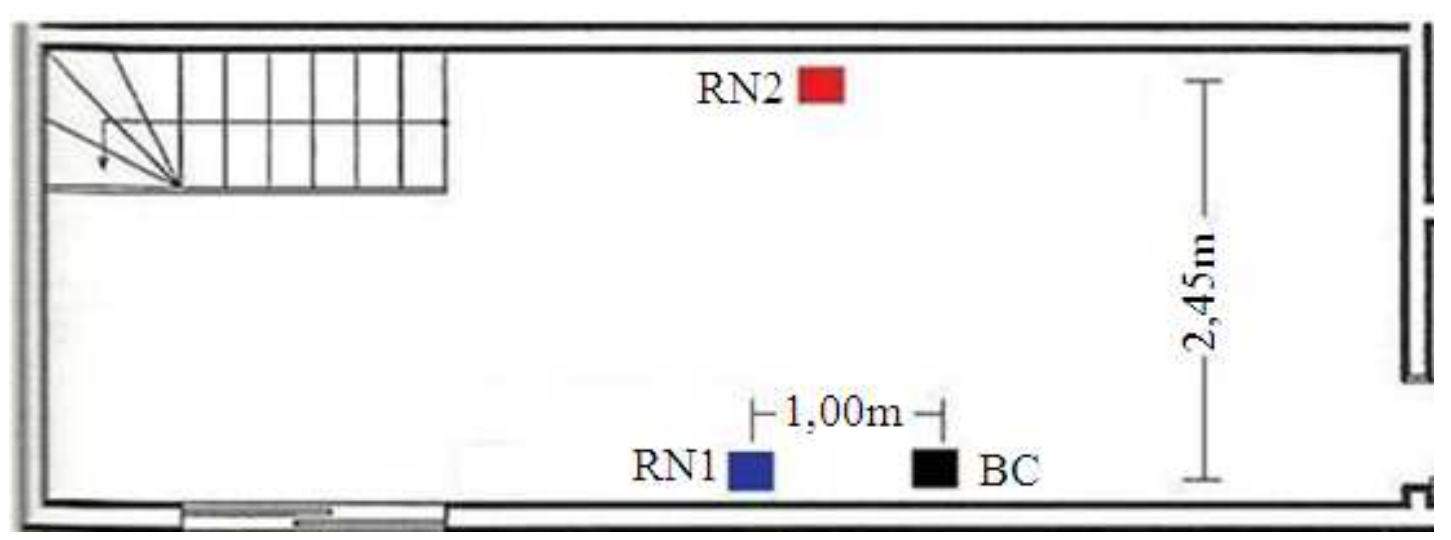

Fig. 3. The room where the data was collected

\section{RESULTS}

Initially, a comparative analysis of the six tested models was conducted to predict the start of battery charge depletion of the sensors in a wireless network. Table 1 presents the results obtained based on the Average Absolute Residues (AAR) of each model.

Considering that the lowest mean absolute residue indicates the model that presents the best fit considering validation data, the first order autoregressive model showed the most appropriate result, with anaverage absolute residue of approximately $0.37 \mathrm{dBm}$, the best result compared to the other models tested in this study. This model is represented by Equation (2):

$$
\mathrm{y}_{\mathrm{t}}=\varnothing_{1} \cdot \mathrm{y}_{\mathrm{t}-1}+\varepsilon_{\mathrm{t}}
$$

whereas: $y_{t}$ corresponds to the RSSI estimate at time $t$, in $\mathrm{dBm} ; \phi_{1}$ corresponds to the single parameter of the first order autoregressive model and $\varepsilon_{t}$ represents a white noise with standard deviation equals to $0,1 \mathrm{dBm}$.

The available data was defined by 700,000 samples, each one collected at every 2 seconds in the WSN during a 16-day period, aiming to stress the batteries and to emphasize the discharge process. These data was divided in two sets (identification and validation set). The last 2000 samples of each set that correspond to battery depletion in the nodes were used in the model determination. The parameter identified in this case was equal to 0,9998845 .

Figure 4 represents the data collected in the RN1 (remote node 1 ).
Table 1. Average residue of the tested models

\begin{tabular}{ll}
\hline Mathematical model & AAR \\
\hline Average & 0,9920907 \\
LinearRegression & 0,8292513 \\
First order autoregressive & 0,3697681 \\
First order autoregressive with a constant & 0,5506384 \\
First order arma & 0,4082996 \\
Second order ARMA & 0,3881594 \\
\hline
\end{tabular}

In this figure, the blue line represents the actual (measured) identification data, the red line represents the validation data and the green line represents the model curve. Figure 5 presents a zoomed window of actual, validation and model data.

Based on both figures, it can be seen that this model can follow the abrupt changes that occur in the battery discharge of the wireless sensor, confirming a good fit between real data and estimated data.

The first failure point caused by battery exhaustion occurred after 16 days and 6 hours and could be clearly detected beforehand by the severe drop in the estimated downlink RSSI, that presented values below $-99 \mathrm{dBm}$.

The chosen model was validated in different situation where the RM2 (remote node 2) sent 4 samples every each second, during almost four days, resulting in 1,350,000 samples collected.

Figure 6 represents the validation with data collected in the RN2 (remote node 2). In this figure, the blue line represents the identification data used for modeling the behavior of RSSI, the red line represents the validation data collected in different conditions than the modeling data and the green line represents the validation curve with new data. Figure 7 presents a zoomed window of the validation made with sampling collected in different conditions of model data. 
Inacio Henrique Yano et al. / Journal of Computer Science 9 (7): 821-826, 2013

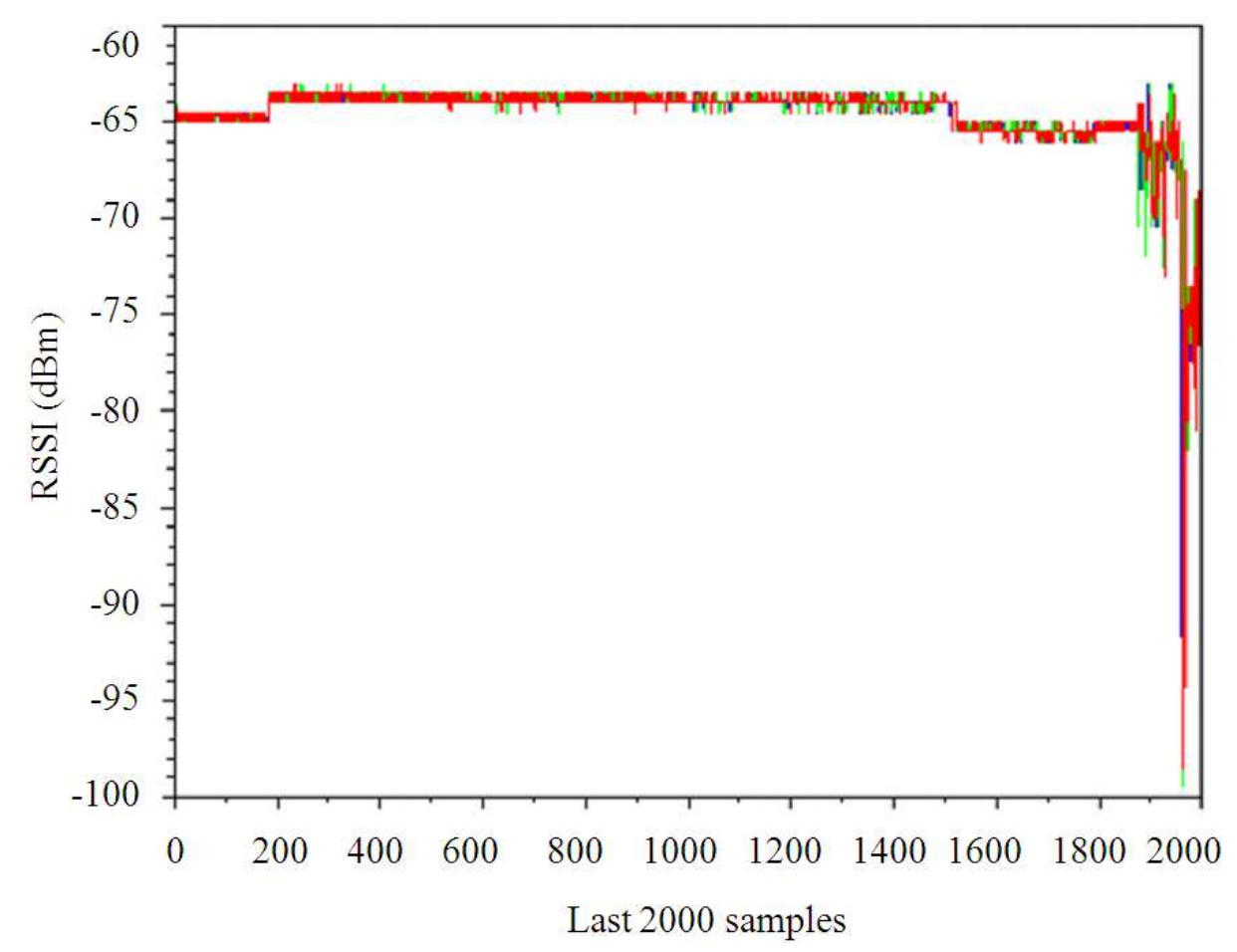

Fig. 4. Results of the autoregressive model

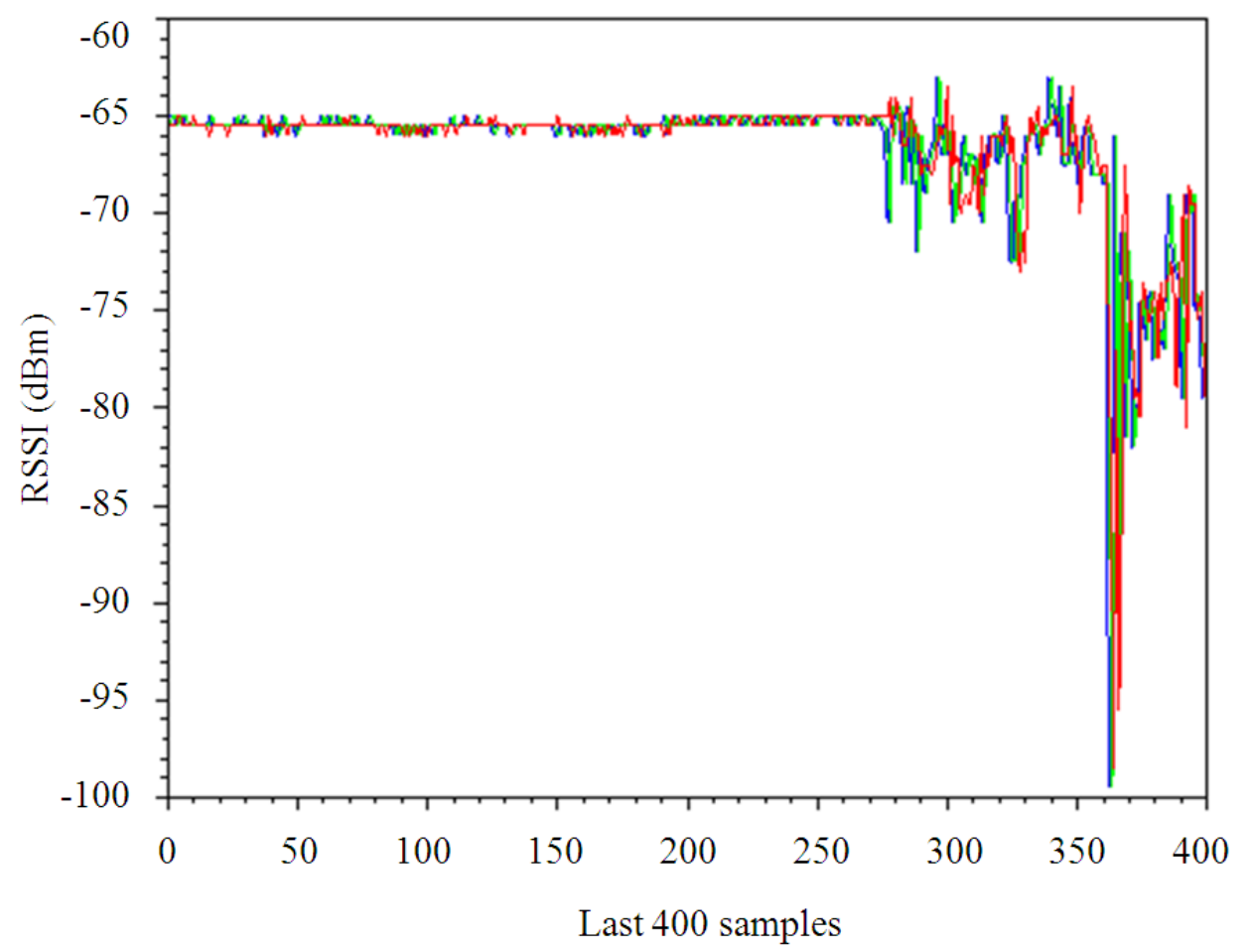

Fig. 5.Visual expansion (zoom) of actual, validation and model data 
Inacio Henrique Yano et al. / Journal of Computer Science 9 (7): 821-826, 2013

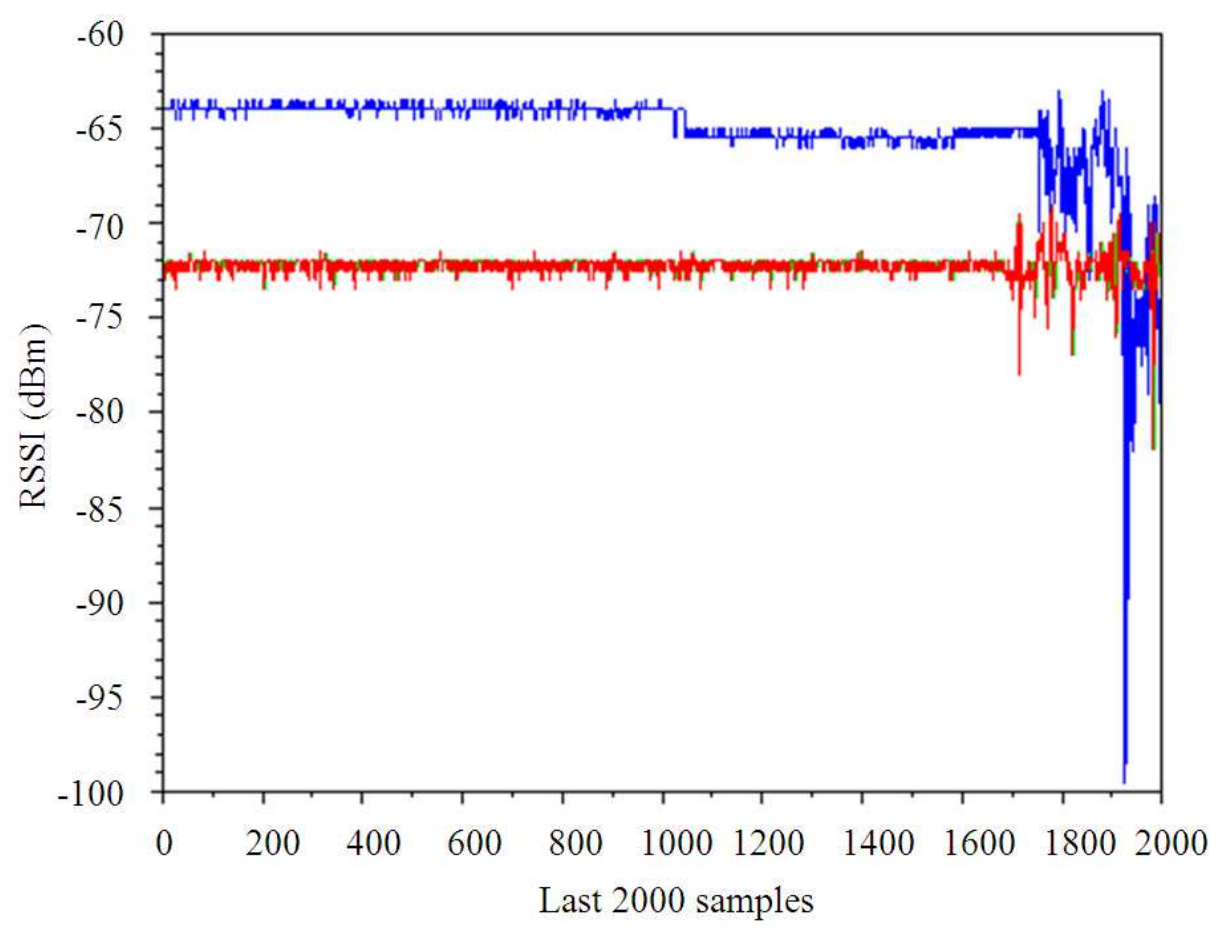

Fig. 6. Results of the Autoregressive Model applied in another data, collected in different conditions

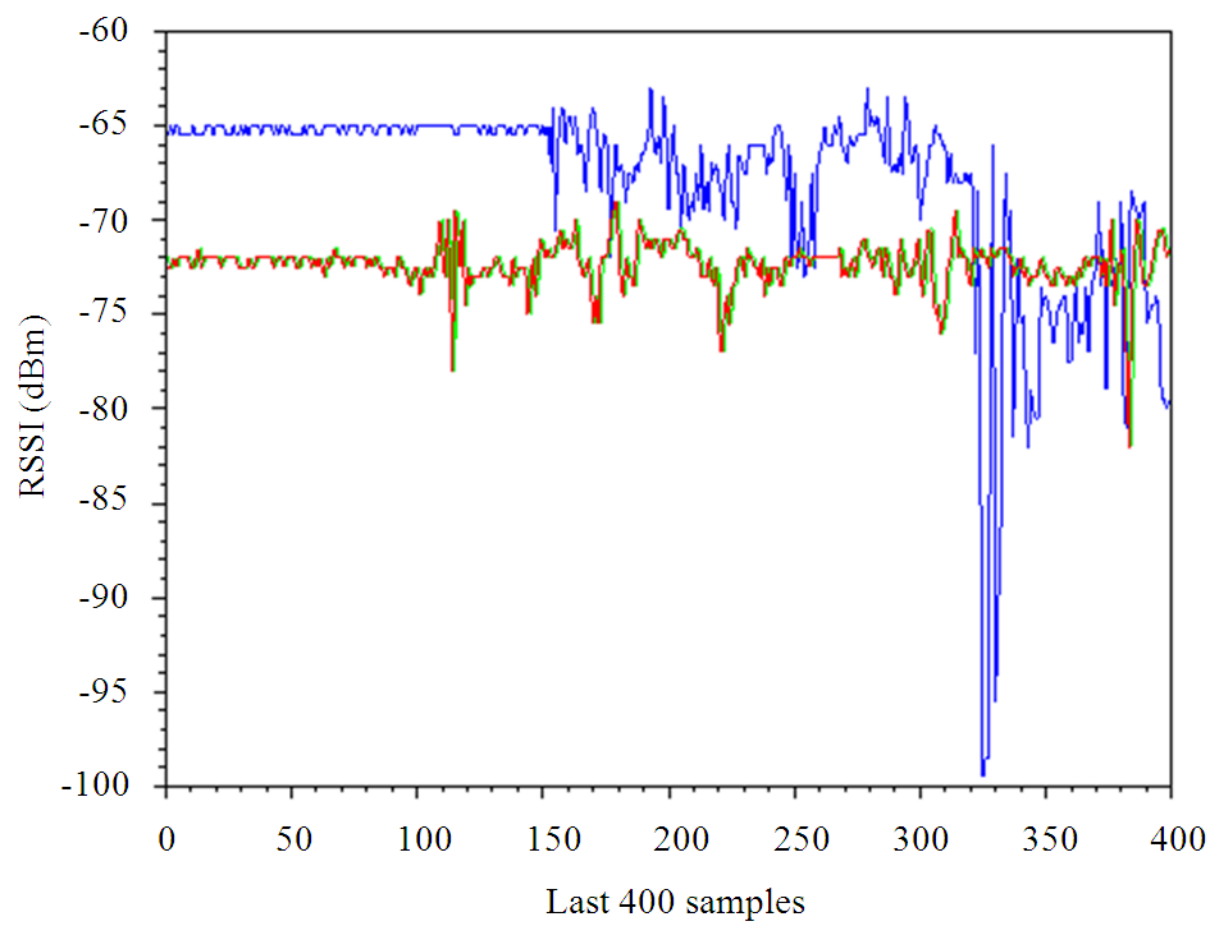

Fig. 7. Visual expansion (zoom) of the validation made with sampling collected in different conditionsof model data (blue) 


\section{DISCUSSION}

According to the obtained results, the model that best represents the depletion of the batteries of a wireless sensor network, based on RSSI samples, was the first order Autoregressive (AR) model.

Even though the behavior and average of the second sampling data were different the identification data, the model curve, represented by the green line, is very tight with the validation curve (red line). The AAR in this second validation (0.3669211) was almost the same AAR obtained in the first validation (0.3697681).

\section{CONCLUSION}

The RSSI can be used to identify battery depletion for static nodes of a Wireless Sensor Network because low level battery charge affects its behavior. But it is important to find the best moment to make the batteries's replacement to avoid data loss with good energy management.

To sum up, with the practical application of systems identification techniques, it was possible to obtain the best mathematical model that reflects the behavior of the WSN nodes RSSI over time, from its startup to the point where batteries depletion occurs.

\section{REFERENCES}

Arduino, 2012a. Analog Reference(type).

Arduino, 2012b. Download the Arduino Software.

ATmega, 2012. 8-bit atmel microcontroller with 4/8/16K bytes in-system programmable flash.

Bourke, P., 2012. AutoRegression Analysis (AR).

Hellan, S. and O. Stengel, 2012. CC1020/1021 received signal strength indicator. Chipcon Products from Texas Instruments.
Kerasiotis, F., A. Prayati, C. Antonopoulos, C. Koulamas and G. Papadopoulos, 2010. Battery lifetime prediction model for a WSN platform. Proceedings of the 4th International Conference on Sensor Technologies and Applications (SENSORCOMM), Jul. 18-25, IEEE Xplore Press, Venice, pp: 525-530. DOI: $10.1109 /$ SENSORCOMM.2010.85

Mafuta, M., M. Zennaro, A. Bagula, G. Ault and H. Gombachika et al., 2012. Successful deployment of a wireless sensor network for precision agriculture in malawi. Proceedings of the 3rd IEEE International Conference on Networked Embedded Systems for Every Application, (NESEA' 12), Liverpool, United Kingdom, pp: 1-26.

Park, P., P.D. Marco, C. Fischione and K.H. Johansson, 2012. Modeling and optimization of the IEEE 802.15.4 protocol for reliable and timely communications. IEEE Trans. Parallel Distribut. Syst., 24: 550-564. DOI: 10.1109/TPDS.2012.159

Raioit, 2012. BE900-Datasheet.

SS, 2013. RFbee V1.1-Wireless arduino compatible node.

TI, 2012. Low-Power Sub-1 GHz RF Transceiver. Texas Instruments.

Tong, B., G. Wang, W. Zhang and C. Wang, 2011. Node reclamation and replacement for long-lived sensor networks. IEEE Trans. Parallel Distribut. Syst., 22: 1550-1563. DOI: 10.1109/TPDS.2011.25

Zhu, M., Y. Xing, H. Zhu and B. Wang, 2012. A new method for communication traffic prediction and anomaly detection. Proceedings of the 8th International Conference on Wireless Communications, Networking and Mobile Computing (WiCOM), Sept. 21-23, IEEE Xplore Press, Shanghai, China, pp: 1-4. DOI: 10.1109/WiCOM.2012.6478317 